\title{
Vaccination Disparity: Quantifying Racial Inequity in COVID-19 Vaccine Administration in Maryland
}

\author{
Stephanie Cardona $(\mathbb{D} \cdot$ Naillid Felipe $•$ Kyle Fischer • \\ Neil Jay Sehgal • Brad E. Schwartz
}

Accepted: 19 May 2021/ Published online: 17 June 2021

(C) The New York Academy of Medicine 2021

\section{Introduction}

As of May 2021, the United States (US) leads in worldwide COVID-19 deaths and infections, with more than 32.5 million confirmed cases and close to 580,000 deaths [1]. This burden, however, has not fallen equally among people in America, and early evidence demonstrates people of color are disproportionately affected by the COVID-19 pandemic [2]. The Maryland Department of Health (MDH) has reported 450,689 COVID19 cases and 8622 deaths as of May 5, 2021, with majority of cases (19\%) occurring in Prince George's (PG) County, a historically Black community [3]. In 2019, the Latinx and Black communities made up $10.4 \%$ and $31 \%$ of Maryland's population respectively [4], yet Black and Latinx are the two leading ethnic demographics with respect to total COVID-19 infections in Maryland [3].

S. Cardona $(\bowtie) \cdot$ N. Felipe

University of Maryland Medical Center, 22 South Greene Street, Suite T3N45, Baltimore, MD 21201, USA

e-mail: scardona@som.umaryland.edu

K. Fischer · B. E. Schwartz

University of Maryland School of Medicine, Baltimore, USA

N. J. Sehgal

University of Maryland School of Public Health, College Park, USA

B. E. Schwartz

University of Maryland Capital Region Health, Lake Arbor, USA

The emergence of a vaccine presents a critical opportunity to provide protection to these vulnerable groups. In December 2020, Maryland began its initial vaccination phase throughout the state. As a result, this study aims to examine the degree to which the initial state vaccine distribution program was concordant to the racial burden of disease.

\section{Methods}

Study Design, Setting, and Population

Descriptive study of COVID-19 cases, deaths, and vaccination rates in 10 Maryland counties with the highest incidence of COVID-19 infection and deaths. County population estimates as of July 1, 2019, as well as demographic data were retrieved from publicly available United States Census Bureau (USCB) data [4]. Data on the proportion of COVID-19 cases, deaths, and first dose vaccination (Pfizer and Moderna) per county were retrieved from the MDH Maryland COVID-19 Data Dashboard [3].

The 10 Maryland counties described in order of highest to lowest incidence of COVID-19 are PG, Montgomery, Baltimore, Baltimore City, Anne Arundel, Washington, Frederick, Harford, Charles, and Howard County. All of the counties are predominantly urban, except for Baltimore city, which is entirely urban with zero percent of its population living in rural areas. Baltimore city also holds the highest population per square mile (7671.5 versus the lowest of 320.2 in 
Charles County) and the highest percentage of persons in poverty $(20.4 \%$ versus the lowest of $5.0 \%$ in Howard County) [4]. The top 5 counties have population estimates greater than 500,000, with PG and Montgomery county approximating a population estimate of one million. The remainder of the described counties have population estimates greater than 150,000 [4].

Categorical variables (race) were reported as percentages and continuous variables (COVID-19 cases, deaths, and population vaccinated) were reported as proportions. Risk ratios were calculated to measure the association between proportion of deaths and proportion of population vaccinated, termed the "vaccination disparity"; a higher ratio indicates greater disparity. We assumed a zero percent mortality from COVID-19 among the population successfully vaccinated $[5,6]$.

\section{Outcomes}

Our outcome of interest is the proportion of deaths to the population vaccinated, termed "vaccination disparity," per county.

\section{Results}

Outcomes

\section{Vaccination Disparity}

PG County has the highest vaccination disparity (1.82), which is 1.6 times higher than the second county with highest incidence of COVID-19 cases, Montgomery (1.09). PG's vaccination disparity was 13 times higher than that calculated in Howard county (0.14), which is the county with the lowest vaccination disparity $(0.14$; Fig. 1).

\section{COVID-19 Cases and Deaths}

The top two counties with the highest proportion of cases (PG) and deaths (Montgomery) were also the counties with the highest proportion of Black and Latinx populations respectively (Fig. 1).

\section{Vaccination and Sociodemographic Factors}

Howard County, with a White population of approximately $50 \%$, an elder population (> 65-year-old) of $14.3 \%$, has the second lowest proportion of deaths $(2.8 \%)$, and yet the highest proportion of population vaccinated (19.5\%) compared to other analyzed counties (Fig. 1). Howard County also holds the highest household income of all the analyzed counties $(\$ 121,160)$ and the lowest percentage of persons in poverty $(5.0 \%)$ [4].

PG County, with a predominantly Black (64.4\%) population and the second highest Latinx (19.5\%) population, had the highest proportion of COVID-19 cases (19.1\%), second highest proportion of deaths (16.1\%), and the lowest proportion of population vaccinated (9.3\%; Fig. 1).

Two out of the three counties least affected by COVID-19 had predominantly White populations (Fig. $1)$.

\section{Discussion}

The COVID-19 pandemic continues to reflect and exacerbate racial inequities in health. Latinx and Black people have been disproportionately affected with higher rates of infection, complications, and mortality compared to White people [7, 8]. This trend remains consistent in Maryland at the county level, with PG County most afflicted by the COVID-19 pandemic, while simultaneously holding the highest percentage of Black residents and the second highest percentage of Latinx residents in the entire state. Furthermore, PG experiences high rates of county members without insurance, and the largest proportion of people without a high school degree [4]. On the contrary, Washington County, a predominantly White county with the lowest median household income, holds the second lowest incidence of COVID-19 cases within the analyzed counties [4].

Given the high infection and mortality rates seen in communities of color, targeted vaccination strategies hold the potential to address racial disparities. Unfortunately, this has not been the case. Maryland began vaccination efforts on December 15, 2020; the vaccination program was divided into separate phases per CDC recommendations, starting with (1) healthcare workers and first responders, (2) individuals aged 75 or older, 


\section{Vaccination Disparity in Maryland}

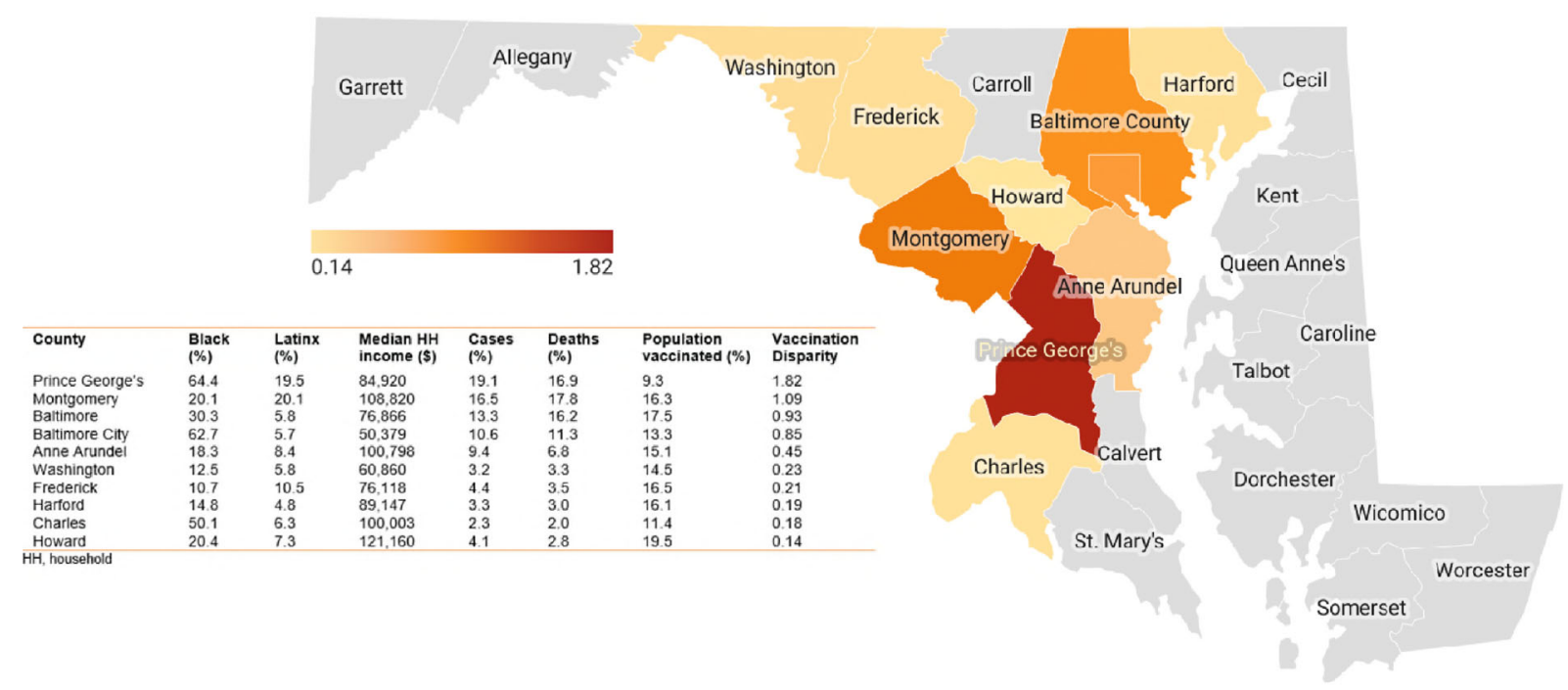

Fig. 1 Sociodemographic factors and vaccination disparity in top 10 Maryland countries with highest incidence of COVID-19 cases and deaths as of March 2021

and those with significant comorbidities, and (3) healthy middle age and young adults [9]. Public data by MDH shows a similar allocation of first-dose vaccines to both PG and Howard counties, with proportionate number of first doses to county's population size $(14.5 \%$ and $15.3 \%$, respectively) [10]. There was a higher number of vaccines distributed to the local health department in Howard $(51.8 \%)$ than in PG County $(30.3 \%)$ and a higher number distributed to Federal Qualified Health Care Centers (FQHCs) in PG $(12.8 \%)$ than in Howard County (2.2\%) [10]. People residing in both counties also had the opportunity to register at one of the four mass-vaccination sites offered in the state (one located in PG County, two located in Baltimore City County, and one located in Charles County) [10].

Despite the similarity in vaccine allocation within these two counties, as of March 5, 2021, PG County, the county with the highest incidence of COVID-19 cases, holds the lowest vaccination rate and the highest vaccination disparity among the state's 10 most affected counties. Alternatively, Howard County, an affluent county with a predominantly White population, achieved the highest percentage of vaccinations despite its relatively small number of COVID-19 cases and deaths - thus making it the county with the lowest vaccination disparity.
One outlier in the study is Charles County, which has a predominantly Black population, and experienced the lowest number of COVID-19 cases and deaths, and has the second lowest vaccination disparity of the counties analyzed. Nonetheless, the population generally holds high levels of household income and education [4], which is likely reflective of how social determinants of health, in this case financial and educational, intersect with race in the COVID-19 pandemic. Furthermore, the low vaccination disparity in Charles County appears driven by low mortality rates rather than disproportionate vaccination. The results observed in Charles County support the need to also center vaccination efforts on other social determinants of health such as education and income.

\section{Structural Racism}

Despite similar vaccine distribution and available vaccination sites in the described counties, different outcomes occurred. The first rollout of Maryland's vaccination program stands as yet another example of the insidious ability of structural racism to impede even the best of public health programs. In isolation, the CDC's phased approach to vaccination is likely both effective and ethical. However, as seen in Maryland during the 
early phases of the vaccine rollout, they may have the potential to exacerbate racial inequities in healthcare access and delivery.

Unfortunately, based on historical trends, phase 1 disproportionately favors vaccination eligibility to White populations and higher socioeconomic groups with longer life expectancies. For example, healthcare workers, residing in phase $1 \mathrm{a}$, are well documented to be disproportionately White [11]. Phase $1 \mathrm{~b}$ includes those who are 75 and older among other qualifying demographics. However, research shows racial disparities in life expectancies between White and Black populations [12]. For example, in Maryland, life expectancies are 80 and 76.9 years for White and Black residents, respectively [13].

\section{Mistrust, Misinformation, and Vaccine Hesitancy}

People of color have mistrust in the medical community due to historical and ongoing discrimination in day-today interactions, as well as high-profile racial injustices, including unconsented and unethical medical experimentation and research practices [14]. The significant negative impact science has had on people of color is a likely contributor for vaccine hesitancy.

Momplaisir et al. conducted focus groups in a predominantly Black community and found that there was high hesitancy towards the vaccine due to mistrust in the healthcare system, the fast speed in which the vaccine was developed, and the uncertainty of its side effects [15]. Another study looking at Black Americans with HIV found that $97 \%$ of participants had at least one reason to distrust the vaccine, and the most common reason was due to beliefs of government withholding information [16]. In this group, 50\% of participants believed Black people would not receive the same medical care as other racial groups [16]. These studies also showed that vaccine recommendation from a trusted health care provider and transparency about safety profile may help reduce hesitancy towards the COVID-19 vaccine $[15,16]$. Moreover, a national survey tracking the public's attitude and experience with COVID-19 vaccinations showed that Black and Latinx communities are concerned about the vaccine's cost despite the vaccine being free to everyone; as a result, clarification regarding the fact that the vaccine is free is crucial since this is a factor taken into consideration when deciding whether or not to get vaccinated [17]. It is important to recognize that people of color may be hesitant but not resistant to the vaccine; having candid conversations and continuing to build trust in the medical community can help reduce hesitancy towards the vaccine.

\section{Access and Outreach}

Ensuring equitable access to the COVID-19 vaccine for Black and Latinx communities is essential to lessen the disproportionate impact the pandemic has already had in these groups. People of color are more likely to be uninsured and lack access to healthcare [18]. They are also less likely to have access to technology, and/or private transportation, making it difficult for these communities to get vaccinated [18].

Maryland has developed a vaccination outreach campaign, GoVAX, and a Vaccine Equity Task Force (VETF), to expand access to underserved and vulnerable communities [19]. These entities have relied on different strategies to achieve their goal, including sound trucks to provide information about COVID-19 prevention and vaccine availability in both English and Spanish, and have also partnered up with non-profit organizations, faith-based entities, and trusted leaders within these communities to increase vaccination among these groups [19]. Furthermore, the state has created telephone-based support lines and appointment systems to help those that do not have access to technology [17]. As discussed, and despite all the aforementioned efforts by the State of Maryland, increased efforts targeting structural racism, communicating and educating our community, expanding outreach campaigns, and ensuring access to healthcare and vaccines are needed in order to narrow the vaccination disparity in communities of color in the State of Maryland.

\section{Limitations}

The counties' data reported was for the overall population and not specific to race. Additionally, we did not control for healthcare workers within the counties which would have affected vaccination rates. We also did not control for the quantity or type of vaccination centers available per county. We assumed a zero percent mortality from COVID-19 among the population vaccinated according to the available literature on COVID-19 vaccine trials. 


\section{Conclusion}

With respect to COVID-19, counties in Maryland with predominantly Black and Latinx populations generally experience higher infection, morbidity and mortality, and lower vaccination rates when compared to counties with predominantly White populations. Deliberate strategies to combat structural racism and vaccine hesitancy among other social determinants of health in Black and Latinx communities are needed to narrow the current racial vaccination disparity.

Acknowledgments The authors received no funding for the work of this manuscript.

\section{References}

1. Johns Hopkins University and Medicine. COVID-19 map. Johns Hopkins Coronavirus Resource Centre. https://coronavirus.jhu.edu/map.html. Accessed 04 Apr 2021.

2. Centers for Disease Control and Prevention. 2020. COVID19 racial and ethnic health disparities. [online] Available at: $<$ https://www.cdc.gov/coronavirus/2019ncov/community/health-equity/racial-ethnicdisparities/index.html. Accessed 01 Mar 2021.

3. Maryland Department of Health. Maryland COVID 19 Data Dashboard. https://coronavirus.maryland.gov/. Accessed 04 Apr 2021.

4. United States Census Bureau. https://www.census. gov/quickfacts/fact/table/princegeorgescounty maryland/PST045219\#PST045219. Accessed 04 May 2021

5. Baden LR, Sahly El HM, Essink B, et al. Efficacy and safety of the mRNA-1273 SARS-CoV-2 vaccine. $N$ Engl J Med. December 2020;384:403-16. https://doi.org/10.1056 /NEJMoa2035389.

6. Polack FP, Thomas SJ, Kitchin N, Absalon J, Gurtman A, Lockhart S, et al. Safety and efficacy of the BNT162b2 mRNA COVID-19 vaccine. $N$ Engl J Med. 2020;383(27): 2603-15. https://doi.org/10.1056/NEJMoa2034577.

7. Yancy CW. COVID-19 and African Americans. JAMA. 2020;323(19):1891-2. https://doi.org/10.1001 /jama.2020.6548.

8. Martinez DA, Hinson JS, Klein EY, Irvin NA, Saheed M, Page KR, et al. SARS-CoV-2 positivity rate for Latinos in the Baltimore-Washington. DC Region JAMA. 2020;324(4): 392-5. https://doi.org/10.1001/jama.2020.11374.

9. Maryland's Phased COVID-19 Vaccine Distribution. https://covidlink.maryland.gov/content/wp-content/uploads/2021 /01/Maryland\%E2\%80\%99s-Phased-COVID-19-VaccineDistribution_1.15.pdf. [Accessed 1 March 2021]

10. County Allocation Report Weeks 1-15. Maryland Department of Public Health; 2021. https://phpa.health.
maryland.gov/Documents/County $\% 20$ Allocation $\% 20$ Report\%20Weeks\%201-15\%20-\%20vMarch29th2021. pdf. Accessed May 4, 2021.

11. U.S. Department of Health and Human Services, 2017. Sex, Race, and Ethnic Diversity of U.S. Health Occupations (2011-2015). [ebook] U.S. Department of Health and Human Services Health Resources and Services Administration Bureau of Health Workforce National Center for Health Workforce Analysis. Available at: $<$ https://bhw.hrsa.gov/sites/default/files/bureau-healthworkforce/data-research/diversity-us-health-occupations. pdf> [Accessed 1 March 2021].

12. Arias E, Tejada-Vera B. and Ahmad F, 2021. Provisional Life Expectancy Estimates for January through June, 2020. 10th ed. [ebook] U.S. Department of Health and Human Services, Centers for Disease Control and Prevention. Available at: <https://www.cdc.gov/nchs/data/vsrr/VSRR10-508.pdf>. Accessed 07 Mar 2021.

13. Hogan L, Rutherford B, Neal R, and Lee Hurt S, 2018. MARYLAND VITAL STATISTICS ANNUAL REPORT 2018. [ebook] Maryland Department of Health Vital Statistics Administration. Available at: <https://health. maryland.gov/talbotcounty/Documents/2018\%20 Maryland\%20Vital\%20Statistics\%20Annual\%20Report. pdf>. Accessed 01 Mar 2021.

14. Team CE. Science has a racism problem. Cell. 2020;181(7): 1443-4. https://doi.org/10.1016/j.cell.2020.06.009.

15. Momplaisir F, Haynes N, Nkwihoreze H, Nelson M, Werner RM, Jemmott J. Understanding drivers of COVID-19 vaccine hesitancy among Blacks [published online ahead of print, 2021 Feb 9]. Clin Infect Dis. 2021;ciab102. doi: 10.1093/cid/ciab102

16. Bogart LM, Ojikutu BO, Tyagi K, Klein DJ, Mutchler MG, Dong L, et al. COVID-19 related medical mistrust, health impacts, and potential vaccine hesitancy among Black Americans living with HIV. J Acquir Immune Defic Syndr. 2021;86(2):2007. https://doi.org/10.1097/QAI.0000000000002570.

17. Ndugga N, Artiga S, Pham O. How are states addressing racial equity in COVID-19 vaccine efforts?. KFF; 2021. https://www.kff.org/racial-equity-and-health-policy/issuebrief/how-are-states-addressing-racial-equity-in-covid-19vaccine-efforts/. Accessed 03 May 2021.

18. Double Jeopardy: COVID-19 And behavioral health disparities for black And Latino communities in the U.S.. U.S. Department of Health \& Human Services; 2021. https://www.samhsa.gov/sites/default/files/covid19behavioral-health-disparities-black-latino-communities.pdf. Accessed May 7, 2021.

19. Maryland Department of Health, 2021. Maryland Equity Task Force Operation Plan. [ebook] Available at: $<$ https://governor.maryland.gov/wp-content/uploads/2021 /03/Vaccine-Equity-Task-Force-Operations-Plan.pdf> [Accessed 10 March 2021].

Publisher's Note Springer Nature remains neutral with regard to jurisdictional claims in published maps and institutional affiliations. 